\title{
Undetected Dural Leaks Complicated by Accidental Drainage of Cerebrospinal Fluid (CSF) can Lead to Severe Neurological Deficits
}

\section{Intrakranielle Hypotension und schwere neurologische Defizite nach akzidenteller Drainage von Liquor bei zuvor undetektierten Duraver- letzungen}

Authors

Affiliation
P. B. Sporns, W. Schwindt, C. D. Cnyrim, W. Heindel, T. Zoubi, S. Zimmer, U. Hanning, T. U. Niederstadt

Department of Clinical Radiology, University Hospital Münster, Germany

\author{
Key words \\ - dural tear \\ - accidental drainage \\ - negative pressure suction \\ - overdrainage \\ - cranial hypotension
}

received $\quad 29.10 .2015$

accepted $\quad 7.12 .2015$

\section{Bibliography}

Dol http://dx.doi.org/ 10.1055/s-0035-1567034

Published online: 4.2.2016

Fortschr Röntgenstr 2016; 188:

451-458 @ Georg Thieme

Verlag KG Stuttgart · New York . ISSN 1438-9029

\section{Correspondence}

Dr. med Peter Bernhard Sporns Institut für Klinische Radiologie, Universitätsklinikum Münster Albert-Schweitzer-Campus 1

Gebäude A1

48149 Münster

Germany

Tel.: ++49/251/8347301

Fax: ++49/2 51/8349656

peter.sporns@hotmail.de

\section{Zusammenfassung \\ $\nabla$}

Ziel: Ausgeprägte intrakranielle Hypotension wurde bereits mehrfach als Komplikation akzidenteller Liquordrainage nach chirurgischen Eingriffen beschrieben. Der Einsatz von Vakuum-basierten Drainagesystemen (Wunddrainagen, VAC®-Wundsystemen, Thoraxdrainagen) führte in diesen Fällen zu lebensbedrohlichen Komplikationen wie intrakraniellen Blutungen und zerebraler Herniation. Im vergangenen Jahr konnten die Autoren 2 Fälle mit akzidenteller spinaler Drainage von Liquor diagnostizieren. Beide Patienten zeigten schwere neurologische Defizite, welche nach Entfernung des Sogs der Drainage komplett rückläufig waren. Material und Methoden: Systematische Recherche in der Datenbank PubMed im Zeitraum 1. Januar 1980 - 1. Oktober 2015 zur Erfassung der Häufigkeit und Varietät der beschriebenen Symptome.

Ergebnisse: Die Literaturrecherche erbrachte 24 Fallberichte mit insgesamt 27 Fällen von posttraumatischem oder postoperativem Liquorverlust, welche zu neurologischen Defiziten führten. Die 15 Patienten, bei denen Drainagen mit Unterdruck verwendet wurden, zeigten schwere neurologische und radiologische Symptome wie Koma, zerebrale Herniation und intrakranielle Blutungen. Bei allen Patienten waren die Symptome nach Entfernung des Sogs der Drainagen schnell rückläufig. Bei den Patienten ohne Applikation von Unterdruck-Drainagen zeigten sich vorwiegend leichtere Symptome, wie Kopfschmerzen und Irritationen von Hirnnerven. Zusätzlich gibt diese Arbeit einen Überblick über aktuelle Empfehlungen zur kranialen und spinalen Bildgebung bei Verdacht auf intrakranielle Hypotension und zum Ausschluss spinaler Liquorleckagen.

Schlussfolgerung: Undetektierte Liquorleckagen kompliziert durch akzidentelle postoperative/ posttraumatische Drainage von Liquor können zu schweren neurologischen Defiziten bei den betroffenen Patienten führen. Die zunehmende Ver-

\section{Abstract \\ $\nabla$}

Purpose: Intracranial hypotension has been reported as a complication of accidental drainage after surgical treatment in several cases. Application of negative pressure systems (wound drains, $\mathrm{VAC}^{\circledR}$-therapy, chest tube drainage) had typically led to severe intracranial hypotension including intracranial hemorrhage and tonsillar herniation. In the last year the authors observed 2 cases of accidental spinal drainage of CSF in patients with neurological deficits, regressing after reduction of the device suction.

Material and Methods: We conducted a systematic PubMed-based research of the literature to study the variety and frequency of the reported symptoms from 1st of January 1980 until 1st of October 2015.

Results: Reviewing the literature 24 relevant citations including 27 reported cases of posttraumatic or postoperative loss of CSF leading to neurological symptoms were identified. All 15 reported cases in which a negative pressure suction device had been applied showed severe neurological and radiological symptoms such as coma or brain herniation and intracranial hemorrhage. In all cases patients recovered rapidly after removal of the suction device. Milder symptoms were observed in the patients without negative pressure suction, mainly only presenting with headaches or cranial nerve involvement.

Additionally, we give an overview about current recommendations regarding cranial and spinal imaging to rule out dural laceration and cranial hypotension.

Conclusion: Patients with dural laceration complicated by accidental drainage of CSF can present with life-threatening conditions. Increasing use of negative pressure suction devices makes the reported condition an important differential diag- 
wendung von Vakuum-basierten Drainagesystemen führt zu einer hohen Relevanz dieses Krankheitsbildes, welches somit eine wichtige Differentialdiagnose darstellt.

Kernaussagen:

- Undetektierte Liquorleckagen kompliziert durch akzidentelle postoperative/posttraumatische Drainage von Liquor können zu lebensbedrohlichen Symptomen führen.

- Die zunehmende Verwendung von Vakuum-basierten Drainagesystemen führt zu einer hohen Relevanz der Differentialdiagnose „Liquorunterdrucksyndrom“. nosis. A precise radiological examination can help to rule out dural laceration and intracranial hypotension.

Key Points:

- Undetected dural laceration complicated by negative pressure suction drains can induce life-threatening symptoms.

- Increasing use of negative pressure suction devices makes the reported condition an important differential diagnosis for radiologists

Citation Format:

- Sporns PB, Schwindt W, Cnyrim CD et al. Undetected Dural Leaks Complicated by Accidental Drainage of Cerebrospinal Fluid (CSF) can Lead to Severe Neurological Deficits. Fortschr Röntgenstr 2016; 188: 451-458

\section{Introduction \\ $\nabla$}

Intracranial hypotension may ensue spontaneously after dural puncture or accidental (intraoperative) surgical opening of the dura [1 -3]. As a complication of this, several cases of accidental drainage after surgical treatment and application of negative pressure systems (wound drain, VAC ${ }^{\circledR}$-therapy, chest tube drainage) have been reported. Under these circumstances severe intracranial hypotension leading to tonsillar herniation and subdural hemorrhage has been reported [ 4 - 7]. In the last year the authors noticed 2 cases of accidental drainage of CSF in which patients showed neurological deficits which were regressive after reduction of the device suction. In times of increasing use of negative pressure suction devices, the reported condition presents an important differential diagnosis. To the authors' knowledge this is the first review that systematically investigates the frequency and variety of emerging symptoms.

\section{Methods}

An English-language PubMed database search using combinations of the descriptors "intracranial hypotension", "intracranial hypovolemia”, "spinal CSF leak”, "lumbar drain”, "subdural hematoma”, “subdural hygroma”, "tonsillar herniation”, “overdrainage”, “coma”, “suction”, “dural tear”, “accidental drainage” and "cerebrospinal fluid" was conducted regarding the period from $1^{\text {st }}$ of January1980 until $1^{\text {st }}$ of October 2015.

\section{Results}

$\nabla$

Reviewing the literature, 24 relevant citations including 27 reported cases of posttraumatic or postoperative loss of CSF leading to neurological symptoms were identified ( $\bullet$ Table 1 ). All 15 reported cases in which a negative pressure suction device had been applied showed severe neurological symptoms. Milder symptoms were observed in the 10 patients without negative pressure suction. In 2 patients it could not be clearly determined from the literature whether a suction drain had been applied.

\section{Etiology}

Fractures of the skull base [8] and the spine [9] can cause traumatic dural tears. In addition spontaneous CSF leaks may occur in approximately $5 / 100000$ patients $[2,3,10,11]$. If the loss of CSF exceeds its production, intracranial hypotension may ensue $[2,9]$. Traumatic dural tears mostly occur after severe spinal trauma. In a retrospective observational study $13 \%$ of all patients with traumatic cervical spine injury featured a dural tear [12]. Patients with initial poor neurological state and disruption of the ligamentum flavum were more often affected [11]. Likewise, $25 \%$ of lumbar burst fractures exhibited dural tears; all of these patients showed a neurological deficit [12]. Despite the dural tear, none of the studied patients exhibited critical intracranial hypotension [13].

\section{Clinical Presentation}

Clinical presentation of intracranial hypotension is heterogenous [1]. Typical neurological symptoms of CSF overdrainage include nausea, emesis, diplopia, orthostatic headaches, dizziness, difficulties in hearing, visual blurring $[2,10]$ and cranial nerve palsy $[14,15]$. Current publications report on reversible coma, intracranial vasospasms [17] or even stroke and death due to intracranial hypotension [11].

After a loss of CSF of around $10 \%$ a dilatation of cerebral sinuses, a sagging of the brain and therefore tension on sensible vessels, nerves and meninges leads to headaches [18].

\section{Reported complications by application of negative pressure suction devices \\ Postoperative wound drains}

Several reports of patients with severe neurological symptoms after spinal surgery and application of suction drains exist [5, $16,20-24]$. An overview of a few cases of postsurgical intracranial hypotension after mainly cranial and only in 2 cases spinal surgery reports about similar symptoms after opening of the dura [19]. Of sixteen patients who remained unconscious or did not become fully responsive after surgery, 4 died and 2 showed brain stem signal intensity changes in MRI. All patients had rapid intraoperative/postoperative CSF loss documented on CT and/or MR imaging studies. The authors came to the conclusion that intracranial hypotension after intracranial and spinal surgery with dural opening is potentially life threatening if patients are left with a subfascial suction drainage.

Similar cases about comatose patients with secondary pseudohypoxic brain swelling after lumbar laminectomy [5] and after thoracic and lumbar spondylodiscitis surgery have been reported [25]. The initial operative procedure in these cases was extradural. In all cases epidural suction drains (Jackson-Pratt drains) were placed in the tissue bed and the initially oriented patients became comatose. Cranial imaging showed subarachnoid hemorrhage and signs suggestive of cerebral anoxia. The fluid from the drain in one case was positive for $\beta-2$ transferrin indicating CSF [5]. This patient fully returned to baseline postoperatively, MRI normalized [5]. The radiographic findings in all cases were re- 
Table 1 Patients with postsurgical and posttraumatic intracranial hypotension.

Tab. 1 Patienten mit postoperativer und posttraumatischer intrakranieller Hypotension.

\begin{tabular}{|c|c|c|c|c|c|c|}
\hline $\begin{array}{l}\text { posttraumatic| } \\
\text { postoperative }\end{array}$ & $\begin{array}{l}\text { suction/ } \\
\text { device }\end{array}$ & symptoms & imaging signs & therapy & outcome & author \\
\hline $\begin{array}{l}\text { postoperative, brachial } \\
\text { plexus avulsion and } \\
\text { reconstructive surgery }\end{array}$ & $\begin{array}{l}\text { pleural } \\
\text { drain }\end{array}$ & $\begin{array}{l}\text { postural headaches, } \\
\text { headaches wors- } \\
\text { ened after aspira- } \\
\text { tion of the pleural } \\
\text { effusion }\end{array}$ & $\begin{array}{l}\mathrm{CT} \text { : tonsillar hernia- } \\
\text { tion and syringomye- } \\
\text { lia } \mathrm{C} 1 \text { to } \mathrm{C} 7\end{array}$ & $\begin{array}{l}\text { surgical closure of } \\
\text { the dural tear }\end{array}$ & $\begin{array}{l}\text { headaches immedi- } \\
\text { ately resolved, MRI } 4 \\
\text { months later: resolu- } \\
\text { tion of cerebellar ton- } \\
\text { sil herniation, regres- } \\
\text { sion of the syrinx }\end{array}$ & Scholsem [26] \\
\hline $\begin{array}{l}\text { posttraumatic, isolated } \\
\text { gunshot wound to the } \\
\text { midaxillary line through } \\
\text { the tenth intercostal } \\
\text { space and a complete } \\
\text { spinal cord injury at T12 }\end{array}$ & $\begin{array}{l}\text { pleural } \\
\text { drain }\end{array}$ & $\begin{array}{l}\text { initially oriented, } \\
\text { comatose with } \\
\text { complete absence } \\
\text { of brainstem } \\
\text { reflexes } 3 \text { hours } \\
\text { after admission }\end{array}$ & $\begin{array}{l}\text { MRI: diffuse cerebral } \\
\text { edema, occlusion of } \\
\text { the bilateral posterior } \\
\text { cerebral arteries, } \\
\text { tonsillar herniation }\end{array}$ & none & death & Kalani [6] \\
\hline $\begin{array}{l}\text { postoperative, thoracic } \\
\text { laminectomy/fusion }\end{array}$ & $\begin{array}{l}\text { pleural } \\
\text { drain }\end{array}$ & $\begin{array}{l}\text { severe neurological } \\
\text { deterioration }\end{array}$ & $\begin{array}{l}\text { CT: bilateral subdural } \\
\text { hematomas, tonsillar } \\
\text { herniation }\end{array}$ & $\begin{array}{l}\text { evacuation of subdural } \\
\text { hematomas and bony } \\
\text { decompression of the } \\
\text { foramen magnum with a } \\
\text { cerebellar tonsillectomy }\end{array}$ & $\begin{array}{l}\text { regained full } \\
\text { consciousness }\end{array}$ & Oudeman [23] \\
\hline posttraumatic & $\mathrm{VAC}^{\circledR}$ & $\begin{array}{l}\text { sudden neurological } \\
\text { deterioration, coma }\end{array}$ & $\begin{array}{l}\text { narrowing of basal } \\
\text { cisterns, tonsillar } \\
\text { cerebellar herniation }\end{array}$ & dural patch & $\begin{array}{l}\text { discharge without } \\
\text { neurologic sequelae }\end{array}$ & Sporns [7] \\
\hline $\begin{array}{l}\text { postoperative, decom- } \\
\text { pressive laminectomies } \\
\text { and lumbosacral } \\
\text { arthrodesis L2-S1 }\end{array}$ & $\begin{array}{l}\text { subfascial } \\
\text { wound drains }\end{array}$ & $\begin{array}{l}\text { severe headache, } \\
\text { generalized tonic } \\
\text { clonic convulsion }\end{array}$ & $\begin{array}{l}\mathrm{CT} \text { : acute and chronic } \\
\text { subdural and intraven- } \\
\text { tricular hemorrhages }\end{array}$ & $\begin{array}{l}\text { burr hole over the } \\
\text { right parietal skull }\end{array}$ & recovered completely & Huh [36] \\
\hline $\begin{array}{l}\text { postoperative, posterior } \\
\text { lumbar interbody fusion } \\
\text { L1-L2 }\end{array}$ & $\begin{array}{l}\text { hemovac } \\
\text { drain }\end{array}$ & $\begin{array}{l}\text { severe headache } \\
\text { with nausea and } \\
\text { vomiting }\end{array}$ & $\begin{array}{l}\mathrm{CT} \text { : acute subdural } \\
\text { hematomas }\end{array}$ & dural patch & recovered completely & Jung [37] \\
\hline $\begin{array}{l}\text { postoperative, bilateral } \\
\text { L3-L4 interlaminectomies } \\
\text { and L3-L4 discectomy }\end{array}$ & $\begin{array}{l}\text { hemovac } \\
\text { drain }\end{array}$ & $\begin{array}{l}\text { left side weakness } \\
\text { accompanied with } \\
\text { nausea and severe } \\
\text { headache }\end{array}$ & $\begin{array}{l}\mathrm{CT} \text { : acute cranial epi- } \\
\text { dural hematoma }\end{array}$ & $\begin{array}{l}\text { evacuation of the } \\
\text { hematoma, minor } \\
\text { dural tear was repaired }\end{array}$ & recovered completely & Grahovac [24] \\
\hline $\begin{array}{l}\text { postoperative, Laminect- } \\
\text { omy L4 / L5 }\end{array}$ & $\begin{array}{l}\text { Jackson-Pratt } \\
\text { drain }\end{array}$ & $\begin{array}{l}15 \text { s period of asys- } \\
\text { tole, comatose for } \\
3 \text { days postopera- } \\
\text { tively }\end{array}$ & $\begin{array}{l}\text { CT: traces of subar- } \\
\text { achnoid hemorrhage } \\
\text { and signs suggestive } \\
\text { of cerebral anoxia }\end{array}$ & removal of the drain & $\begin{array}{l}\text { fully recovered on } \\
\text { hospital day } 10 \text {, } \\
\text { MRI on hospital } \\
\text { day } 8 \text { normalized }\end{array}$ & Fehnel [5] \\
\hline $\begin{array}{l}\text { postoperative, Harring- } \\
\text { ton rod placement for } \\
\text { lumbar scoliosis }\end{array}$ & $\begin{array}{l}\text { Jackson-Pratt } \\
\text { drain }\end{array}$ & $\begin{array}{l}\text { abrupt deterioration } \\
\text { in mental status }\end{array}$ & $\begin{array}{l}\text { CT: tonsillar hernia- } \\
\text { tion with superior } \\
\text { cerebellar infarction }\end{array}$ & removal of the drain & $\begin{array}{l}\text { mental status } \\
\text { improved slowly } \\
\text { after removal of } \\
\text { the drain, remained } \\
\text { quadriparetic }\end{array}$ & Andrews [20] \\
\hline $\begin{array}{l}\text { postoperative, cranio- } \\
\text { tomy for resection of } \\
\text { cavernous malformation } \\
\text { with intraoperative } \\
\text { lumbar drainage }\end{array}$ & $\begin{array}{l}\text { lumbar } \\
\text { drain }\end{array}$ & $\begin{array}{l}\text { drowsy, opened } \\
\text { eyes to commands, } \\
\text { oriented only to } \\
\text { name }\end{array}$ & $\begin{array}{l}\mathrm{CT} \text { : cerebellar } \\
\text { tonsillar herniation }\end{array}$ & $\begin{array}{l}\text { lumbar blood patch, } \\
\text { decompressive subocci- } \\
\text { pital craniectomy and } \\
\text { C-1 laminectomy with } \\
\text { duroplasty }\end{array}$ & $\begin{array}{l}\text { lasting neuropathic } \\
\text { pain and cervical cord } \\
\text { signal changes on MRI }\end{array}$ & Sugrue [4] \\
\hline $\begin{array}{l}\text { postoperative, clipping } \\
\text { of a ruptured aneurysm }\end{array}$ & $\begin{array}{l}\text { lumbar } \\
\text { drain }\end{array}$ & $\begin{array}{l}\text { coma, signs of cra- } \\
\text { nial nerves involve- } \\
\text { ment, brain stem } \\
\text { and cerebellar dys- } \\
\text { function }\end{array}$ & $\begin{array}{l}\text { MRI: brain sagging } \\
\text { and cerebellar } \\
\text { tonsillar herniation }\end{array}$ & $\begin{array}{l}\text { epidural blood patch, } \\
\text { ventricular drainage, } \\
\text { and Trendelenburg } \\
\text { position }\end{array}$ & $\begin{array}{l}\text { discharged } \\
\text { without sequelae }\end{array}$ & Bloch [21] \\
\hline $\begin{array}{l}\text { postoperative, lumbar } \\
\text { spinal surgery }\end{array}$ & $\begin{array}{l}\text { lumbar } \\
\text { drain }\end{array}$ & $\begin{array}{l}\text { neurological dete- } \\
\text { rioration, coma }\end{array}$ & $\begin{array}{l}\mathrm{CT} \text { : thrombosis of the } \\
\text { superior sagittal si- } \\
\text { nus, the right trans- } \\
\text { verse sinus, the right } \\
\text { sigmoid sinus, and the } \\
\text { right jugular vein }\end{array}$ & dural patch & recovered completely & $\begin{array}{l}\text { Lourenço Costa } \\
\text { [38] }\end{array}$ \\
\hline $\begin{array}{l}\text { tap test with continued } \\
\text { CSF flow for almost } \\
30 \text { min }\end{array}$ & CSF tap & $\begin{array}{l}\text { sudden neurological } \\
\text { deterioration, pupils } \\
\text { sluggishly reacting } \\
\text { to light and general- } \\
\text { ized tonic-clonic } \\
\text { seizures }\end{array}$ & $\begin{array}{l}\mathrm{CT} \text { : multiple infra- and } \\
\text { supratentorial intra- } \\
\text { cerebral hemorrhages } \\
\text { with irruption into the } \\
\text { ventricular system }\end{array}$ & ventilatory assistance & death & $\begin{array}{l}\text { Ruiz-Sandoval } \\
\text { [39] }\end{array}$ \\
\hline
\end{tabular}


Table 1 (Continuation)

\begin{tabular}{|c|c|c|c|c|c|c|}
\hline $\begin{array}{l}\text { posttraumatic| } \\
\text { postoperative }\end{array}$ & $\begin{array}{l}\text { suction/ } \\
\text { device }\end{array}$ & symptoms & imaging signs & therapy & outcome & author \\
\hline $\begin{array}{l}\text { postoperative, } \\
\text { thoracic spondylo- } \\
\text { discitis surgery }\end{array}$ & $\begin{array}{l}\text { epidural } \\
\text { suction drain }\end{array}$ & $\begin{array}{l}\text { epileptic seizures, } \\
\text { coma }\end{array}$ & $\begin{array}{l}\text { pseudohypoxic } \\
\text { brain swelling }\end{array}$ & removal of the drain & subsequent recovery & Parpaley [22] \\
\hline $\begin{array}{l}\text { postoperative, lumbar } \\
\text { spondylodiscitis surgery }\end{array}$ & $\begin{array}{l}\text { epidural } \\
\text { suction drain }\end{array}$ & $\begin{array}{l}\text { sudden neurological } \\
\text { deterioration, coma }\end{array}$ & $\begin{array}{l}\mathrm{CT} \text { : basal ganglia, } \\
\text { cerebellar and } \\
\text { brainstem infarction }\end{array}$ & removal of the drain & death & Parpaley [22] \\
\hline $\begin{array}{l}\text { postoperative, thoracic } \\
\text { schwannoma resection }\end{array}$ & no & $\begin{array}{l}\text { intermittent head- } \\
\text { aches, right ptosis, } \\
\text { cranial nerve III palsy }\end{array}$ & $\begin{array}{l}\text { CT: small subdural } \\
\text { hematoma } \\
\text { MRI: diffuse leptome- } \\
\text { ningeal enhancement } \\
\text { and crowding of the } \\
\text { foramen magnum }\end{array}$ & conservative treatment & fully recovered & Lau [40] \\
\hline $\begin{array}{l}\text { postoperative, thoracic } \\
\text { disc excision }\end{array}$ & no & $\begin{array}{l}\text { acute diplopia, cra- } \\
\text { nial nerve VI palsy }\end{array}$ & $\begin{array}{l}\text { CT: bilateral subdural } \\
\text { effusion }\end{array}$ & conservative treatment & $\begin{array}{l}\text { recovered completely } \\
\text { in } 3 \text { months }\end{array}$ & Khurana [41] \\
\hline $\begin{array}{l}\text { postoperative, thoracic } \\
\text { disc excision }\end{array}$ & no & $\begin{array}{l}\text { diplopia, occasional } \\
\text { headaches, cranial } \\
\text { nerve VI palsy }\end{array}$ & no & conservative treatment & $\begin{array}{l}\text { recovered completely } \\
\text { over a period of } \\
5 \text { months }\end{array}$ & Khurana [41] \\
\hline $\begin{array}{l}\text { postoperative, micro- } \\
\text { scopic discectomy L5-S1 }\end{array}$ & no & $\begin{array}{l}\text { delayed postural } \\
\text { headache and pho- } \\
\text { tophobia }\end{array}$ & $\begin{array}{l}\text { MRI: enhancement of } \\
\text { the pachymeninges } \\
\text { CT-myelography: } \\
\text { CSF leak }\end{array}$ & $\begin{array}{l}\text { epidural blood patch, } \\
\text { eventually direct suture } \\
\text { plication }\end{array}$ & $\begin{array}{l}\text { discharge without } \\
\text { sequelae }\end{array}$ & Kundu [42] \\
\hline $\begin{array}{l}\text { postoperative, lumbar } \\
\text { surgery for failed back } \\
\text { syndrome }\end{array}$ & no & $\begin{array}{l}\text { expressive aphasia, } \\
\text { headache }\end{array}$ & $\begin{array}{l}\mathrm{CT} \text { : acute subdural } \\
\text { hematoma }\end{array}$ & $\begin{array}{l}\text { evacuation of the } \\
\text { hematoma, operative } \\
\text { dural patch }\end{array}$ & $\begin{array}{l}\text { discharged without } \\
\text { sequelae }\end{array}$ & Sciubba [16] \\
\hline $\begin{array}{l}\text { postoperative, incidental } \\
\text { durotomy }\end{array}$ & no & - & $\begin{array}{l}\text { extensive but } \\
\text { reversible cerebral } \\
\text { vasospasm }\end{array}$ & $\begin{array}{l}\text { operative dural } \\
\text { tear repair }\end{array}$ & $\begin{array}{l}\text { discharged without } \\
\text { sequelae }\end{array}$ & Chaves [17] \\
\hline $\begin{array}{l}\text { postoperative, spinal in- } \\
\text { strumentation surgery } \\
\text { for scoliosis } 3 \text { months } \\
\text { prior to her admission }\end{array}$ & no & headaches & $\begin{array}{l}\mathrm{CT} \text { : thoracic pedicle } \\
\text { screw penetrating } \\
\text { and transversing } \\
\text { the dura mater at } \\
\text { the T3-T4 level }\end{array}$ & $\begin{array}{l}\text { dural patch, revision } \\
\text { of the screw }\end{array}$ & recovered completely & Albayram [43] \\
\hline $\begin{array}{l}\text { postoperative, surgery for } \\
\text { a nerve sheet tumor origi- } \\
\text { nating at the level of the } \\
\text { right second neural root }\end{array}$ & no & $\begin{array}{l}\text { horizontal diplopia } \\
\text { occurred, palsy of } \\
\text { the left abducens } \\
\text { nerve }\end{array}$ & $\begin{array}{l}\text { cranial MRI: diffuse } \\
\text { pachymeningitis } \\
\text { thoracic MRI: pseudo- } \\
\text { meningocele around } \\
\text { the second interver- } \\
\text { tebral foramen }\end{array}$ & $\begin{array}{l}\text { conservative } \\
\text { management }\end{array}$ & $\begin{array}{l}\text { complete recovery } \\
6 \text { months later }\end{array}$ & Bobbio [44] \\
\hline $\begin{array}{l}\text { postoperative, spinal } \\
\text { surgery for severe } \\
\text { myelodysplastic scoliosis }\end{array}$ & no & aphasic, somnolent & $\begin{array}{l}\text { CT: massive subdural } \\
\text { hematoma, pneumo- } \\
\text { cephalus, pneumor- } \\
\text { rachis and a malposi- } \\
\text { tioned pedicular } \\
\text { screw }\end{array}$ & $\begin{array}{l}\text { cranial decompression } \\
\text { and spinal reoperation } \\
\text { with dural tear repair }\end{array}$ & recovered completely & Nowak [45] \\
\hline caudal epidural injection & no & $\begin{array}{l}\text { orthostatic head- } \\
\text { ache with general- } \\
\text { ized weakness and } \\
\text { syncopal episodes }\end{array}$ & no & epidural blood patch & recovered completely & Thomas [46] \\
\hline $\begin{array}{l}\text { postoperative, } \\
\text { spondylodiscitis T6/7 }\end{array}$ & - & - & $\begin{array}{l}\mathrm{CT} \text { : compressed } \\
\text { ambient cisterns, } \\
\text { basal ganglia } \\
\text { hypoattenuation }\end{array}$ & - & recovered completely & Hadizadeh [19] \\
\hline $\begin{array}{l}\text { postoperative, } \\
\text { Spondylodiscitis } L 4 / 5 \text {, } \\
\text { spinal fixation }\end{array}$ & - & - & $\begin{array}{l}\text { MRI: brain stem } \\
\text { and thalamic/basal } \\
\text { ganglia lesions }\end{array}$ & - & death & Hadizadeh [19] \\
\hline
\end{tabular}

No = no drain used, $-=$ no information available.

ported to be most consistent with acute intracranial hypotension relating to acute loss of CSF.

Two patients in the literature presented with neurogenic shock after posterior lumbar interbody fusion (PLIF). One patient showed bradycardia and the other developed cardiac arrest just after the surgical incision was closed and the drainage tube opened. In these cases incarceration of multiple cauda equina rootlets after the accidental dural tear by suction drainage seems to have caused a sudden decrease of cerebrospinal fluid pressure and traction of the cauda equina, which may have led to the vasovagal reflex. This shows that not only cranial but other lifethreatening conditions can occur. 

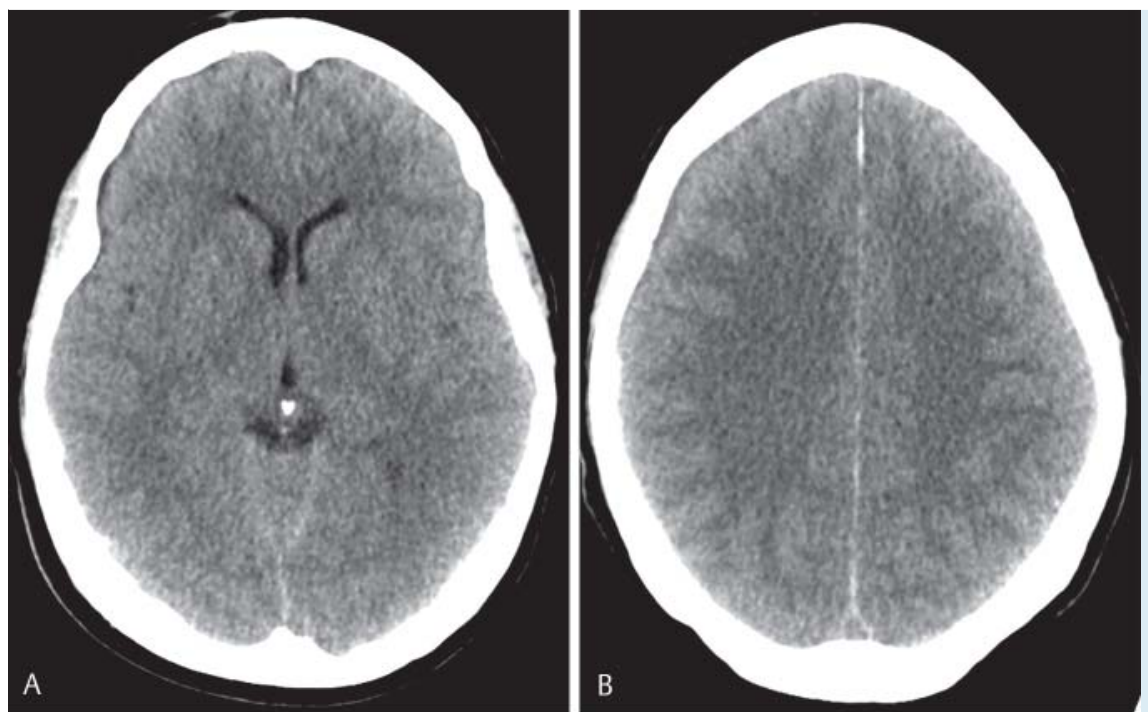

Fig. 1 Previously unreported case of a patient who underwent lumbar stabilization. After application of a suction drain the cranial CT shows a frontal hygroma $\mathbf{A}+\mathbf{B}$ on the right and effacement of the sulci $\mathbf{B}$. After removal of the drain the cranial CT three days later is normal; ventricular size has increased C+D.

Abb. 1 Noch nicht veröffentlichter Fall eines Patienten nach lumbaler, dorsaler Stabilisierung. Nach Anlage einer lumbalen Unterdruckdrainage zeigt das axiale Schädel-CT ein frontales Hygrom rechts $\mathbf{A}+\mathbf{B}$ und ein Verstreichen der Sulci B.Nach Entfernung der Drainage ist das Kontroll-CT 3 Tage später wieder normal, die Ventrikelweite hat wieder zugenommen $\mathrm{C}+\mathrm{D}$.
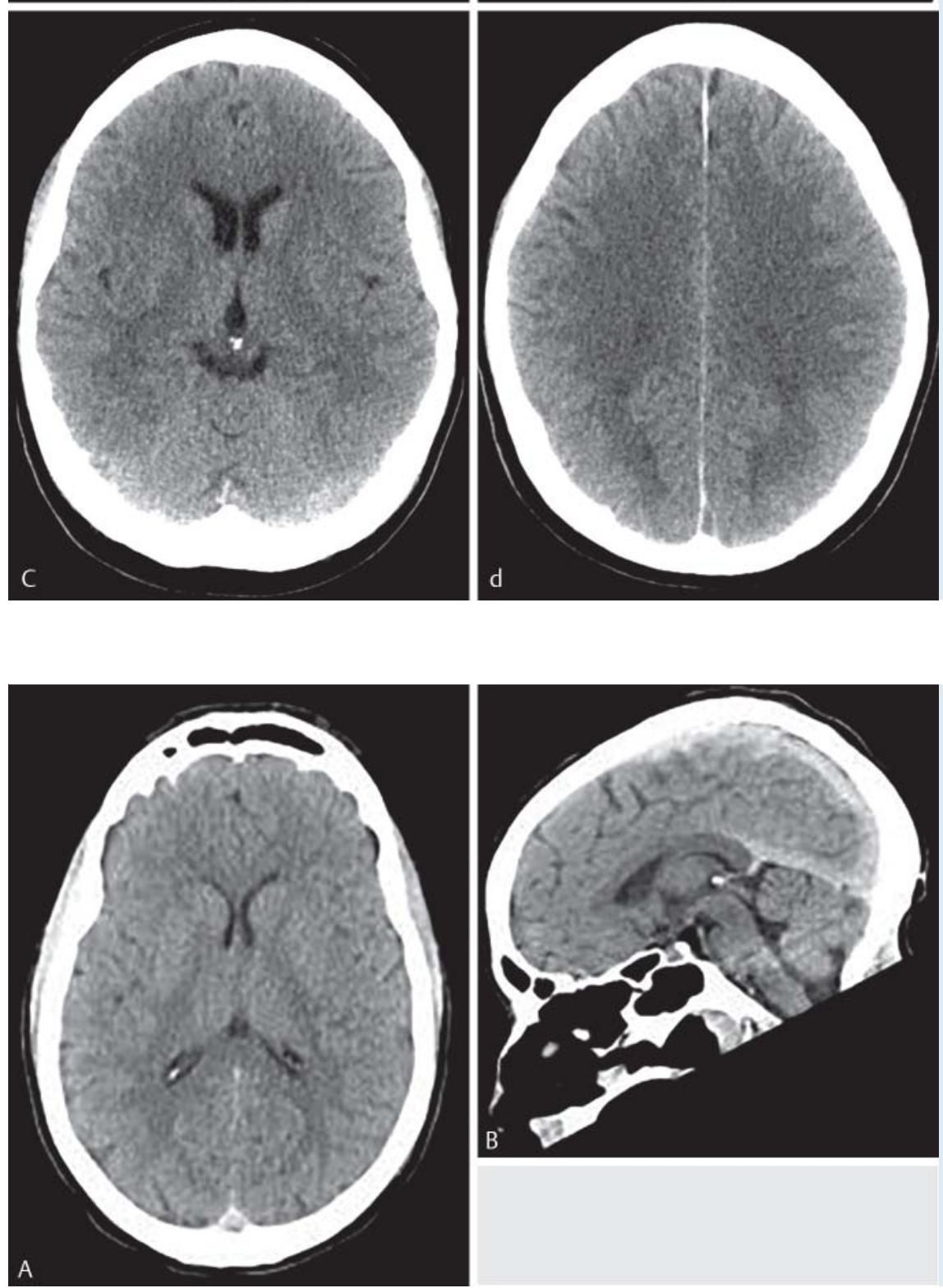

Fig. 2 Previously reported case of a patient who had severe intracranial hypotension after VAC ${ }^{\circledR}$ wound therapy. Computed tomography (CT) of the head before VAC ${ }^{\circledR}$ application; A Head axial and $\mathbf{B}$ Head sagittal MPR showing a normal cranial CT.

Abb. 2 Bereits vorab veröffentlichter Fall eines Patienten mit schwerer intrakranieller Hypotension nach Anlage eines VAC-Wundverbandes. Das CT vor VAC-Applikation ist unaufällig $\mathbf{A}+\mathbf{B}$. 

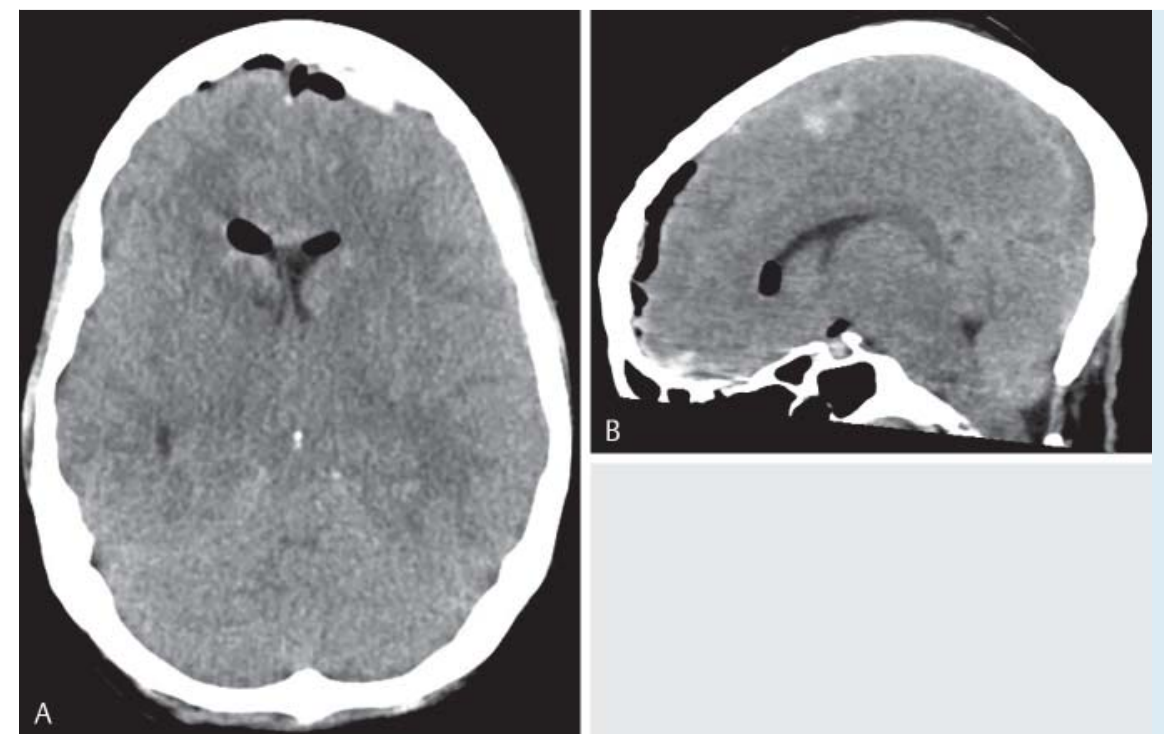

Fig. 3 CT-scan head after $\mathrm{VAC}^{\circledR}$ application; $\mathrm{A}$ axial showing an effacement of the basal cisterns and intracranial/intraventricular air after implantation of an EVD. Note the "slit like" ventricles. B (sagittal MPR) is showing a sagging of the brain with lowering of the cerebellar tonsils (tonsillar herniation).

Abb. 3 Das CT nach VAC-Applikation; A axial zeigt ein Verstreichen der basalen Zisternen und intrakranielle/intraventrikuläre Luft nach Anlage einer EVD. Zudem sind die Ventrikel nur noch schlitzförmig abgrenzbar. B (sagittale MPR) zeigt das Tiefertreten des Hirns mit Verlagerung der Kleinhirntonsillen in das Foramen magnum (Tonsilläre Herniation).

\section{Report of a new Case}

A previously unreported patient underwent lumbar stabilization. A suction drain was applied in the tissue bed and in the postoperative course the patient developed a global aphasia and disorientation. The head CT showed a frontal hygroma on the right and an effacement of the sulci ( $\bullet$ Fig. 1A, B). The drain was removed immediately and the neurological symptoms of the patient fully recovered on the same day. A control CT three days later was normal ( $\bullet$ Fig. 1C, D).

\section{$\operatorname{VAC}^{\circledR}$ therapy}

Recently we have reported about a polytraumatized patient with a life-threatening event of intracranial hypotension after $\operatorname{VAC}^{\circledR}$ wound therapy [7]. This patient had suffered a pelvic fracture after a motor vehicle accident. Initial cranial CT was normal (○ Fig. 2). After application of a VAC ${ }^{\circledR}$ wound dressing, the initially oriented patient became nonresponsive. The following cranial CT showed signs of intracranial hypotension ( $\odot$ Fig. 3). Pneumorrhachis in native CT indicated a dural tear ( $\bullet$ Fig.4) which was confirmed by CT myelography at L5/S1 level ( $\bullet$ Fig. 5) and consequently the $\operatorname{VAC}^{\circledR}$ was removed. After application of a dural patch the patient was oriented post-operatively and the cranial CT improved to a normal state ( $\bullet$ Fig. 6 ).

\section{Chest tube drainage}

Posttraumatic CSF loss with loss of consciousness and tonsillar herniation has been reported as a complication of chest tube drainage after spinal injury [6]. The patient presented to the trauma service awake, alert, and oriented with an isolated gunshot wound leading to a complete spinal cord injury at T12. A chest tube was placed, and the patient was found to be comatose with complete absence of brainstem reflexes 3 hours after admission. MRI and autopsy revealed diffuse cerebral edema, occlusion of the bilateral posterior cerebral arteries, and tonsillar herniation extending several centimeters below the foramen magnum, with petechial hemorrhages and absence of gliosis.

Another case of tonsillar herniation and syringomyelia after brachial plexus avulsion and reconstructive surgery with a cerebrospinal fluid leak between the cervical subarachnoid space and the pleural cavity has been reported [26]. The patient showed a persistent right pleural effusion for about four months

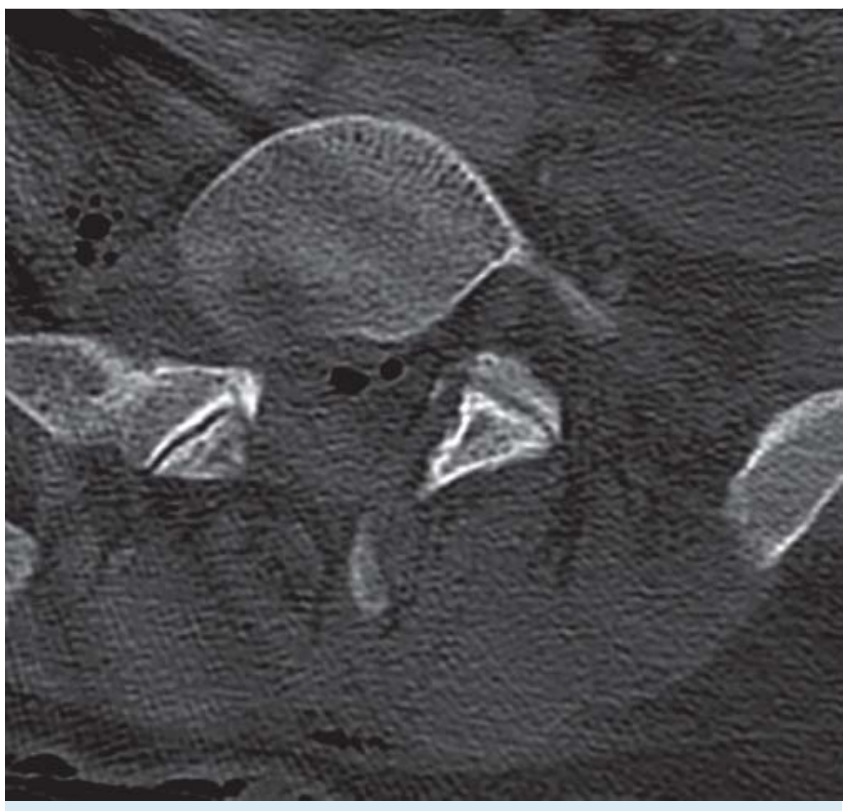

Fig. 4 The axial CT is showing intrathecal air called "pneumorrhachis".

Abb. 4 Das axiale CT zeigt intrathekale Luft; „Pneumorrhachis“.

after reconstructive surgery for a right brachial plexus avulsion. Initially present headaches had worsened considerably after two aspirations of the pleural effusion. MRI demonstrated signs of chronic intracranial hypotension and tonsillar herniation with a presyrinx cavity from vertebral level $\mathrm{C} 1$ to $\mathrm{C}$. Plexus brachial MRI confirmed the presence of a cerebrospinal fluid leak between the avulsed root of C8 and the pulmonary apex. After dural closure, the patient's headaches immediately resolved, and MRI four months later showed resolution of cerebellar tonsillar herniation and regression of the syrinx.

\section{Radiological Examination}

Cranial Imaging

Typical imaging signs of intracranial hypotension which can be depicted in CT and MRI include "slit like" lateral ventricles, subdural fluid collections and effacement of sulci, as well as the downward 

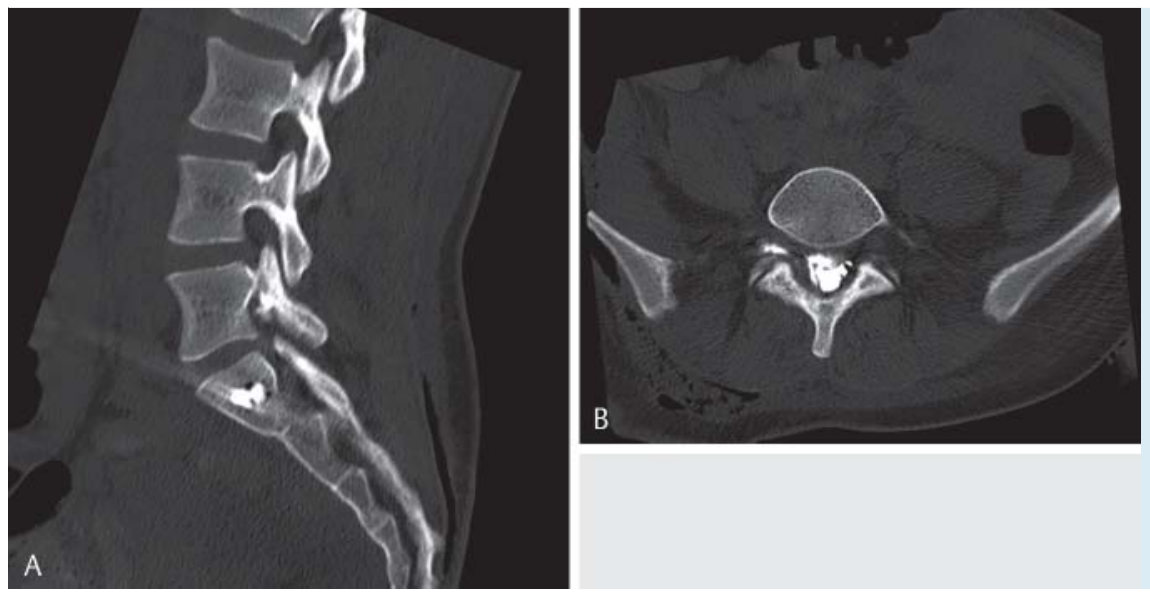

Fig. 5 CT-myelography; A sagittal and B axial MPR show a CSF leak L5 / S1 on the right.

Abb. 5 Die CT-Myelographie; A sagittale und B axiale MPR zeigt ein Liquorleck auf Höhe L5/S1 rechts.

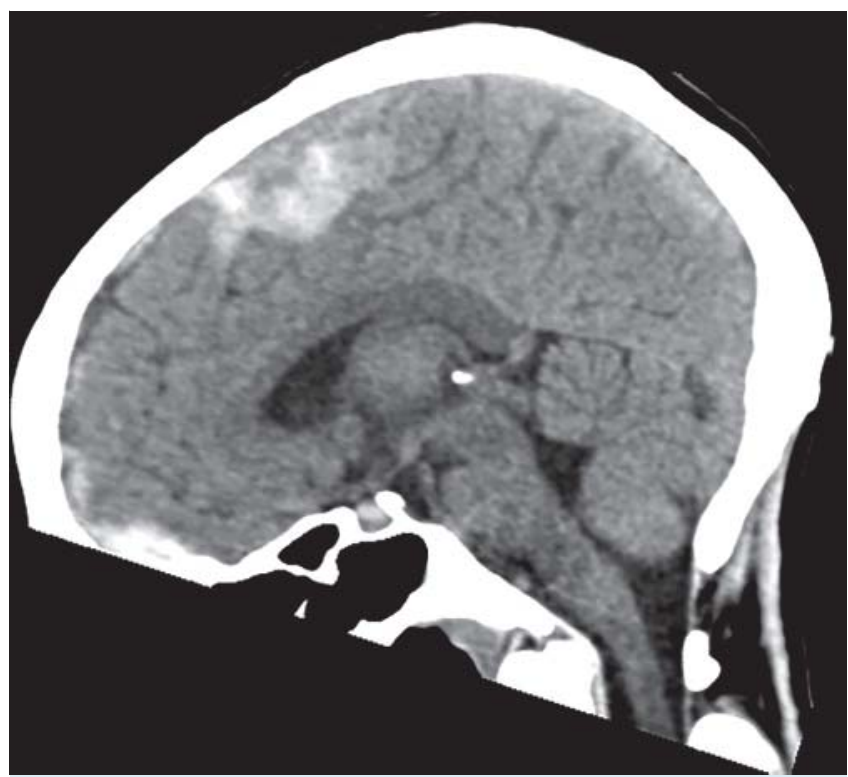

Fig. 6 CT-scan head; the sagittal MPR five days after removal of the VAC shows a regressive lowering of the cerebellar tonsils.

Abb. 6 Das abschließende Schädel-CT fünf Tage nach Entfernung des VAC zeigt eine Zurückverlagerung der Kleinhirntonsillen in die ursprüngliche Position.

transtentorial herniation of the brain with the "sagging brain" appearance $[1,19,27]$. Imaging modality of choice is an MRI of the head. Features include thickening and enhancement of the dura after contrast application, enlargement of venous structures with dilation of the intracranial dural sinuses and spinal epidural plexuses, and engorgement of the pituitary gland $[1,19]$. Moreover hyperintensities in the thalamus/basal ganglia, brain stem, and cerebellum have been reported as a possible result of impaired drainage through the internal cerebral veins and the vein of Galen into the straight sinus $[19,25]$.

Subdural hygromas, often bihemispheric and with an infratentorial predominance, usually are named as a typical imaging finding $[3,19]$. However, they were only found in $30 \%$ of patients and did not correlate with clinical outcome parameters [19].
Spinal Imaging

A possible indication for traumatic dural laceration may be pneumorrhachis, well discernible in CT $[13,28]$. With MRI, dural tears can be detected using fluid-sensitive and fat-suppressive sequences. Typical are fluid collections along the paravertebral tissue and spinal nerves [29]. MRI is the modality of choice for depicting plexus injuries and nerve root avulsions which can be associated with dural laceration [30,31]. For this purpose MR neurography shows first promising results [32, 33]. Using CT or MR myelography with adjunction of intrathecal contrast medium loss of CSF can directly be detected $[7,34,35]$. However, this contains a risk of herniation or postpunctional CSF depletion.

\section{Conclusion}

\section{$\nabla$}

In most of the cases with severe neurological symptoms, in addition to the dural tear, extra negative pressure had been applied. This implies that apart from the dural tear, additional factors such as suction of cerebrospinal fluid are needed to induce severe symptoms. In conclusion, patients with suspected dural laceration complicated by accidental drainage of CSF can present with life-threatening conditions. Increasing use of negative pressure suction devices makes the reported condition an important differential diagnosis so that acute intracranial hypotension should be considered as an explanation of postoperative coma after cranial or spinal surgery. A precise radiological examination can help to rule out dural laceration and intracranial hypotension.

\section{References}

1 Urbach $H$. Intracranial hypotension: Clinical presentation, imaging findings, and imaging-guided therapy. Curr Opin Neurol 2014; 27: 414-424

2 Mokri B. Spontaneous CSF leaks: Low CSF volume syndromes. Neurol Clin 2014; 32: 397-422

3 Hoffmann J, Goadsby PJ. Update on intracranial hypertension and hypotension. Curr Opin Neurol 2013; 26: 240-247

4 Sugrue PA, Hsieh PC, Getch CC et al. Acute symptomatic cerebellar tonsillar herniation following intraoperative lumbar drainage. J Neurosurg 2009; 110: 800-803

5 Fehnel CR, Razmara A, Feske SK. Coma from wall suction-induced CSF leak complicating spinal surgery. BMJ Case Rep 2014; 2014 10.1136/ bcr,2014-203801

6 Kalani MY, Filippidis A, Martirosyan NL et al. Cerebral herniation as a complication of chest tube drainage of cerebrospinal fluid after injury to the spine. World Neurosurg 2013; 79: 798.E17 - 798.E19 
7 Sporns PB, Zimmer S, Hanning $U$ et al. Acute tonsillar cerebellar herniation in a patient with traumatic dural tear and VAC therapy after complex trauma. Spine J 2015; 15: e13-e16

8 Baugnon KL, Hudgins PA. Skull base fractures and their complications. Neuroimaging Clin N Am 2014; 24: 439 - 465, vii-viii

9 Luszczyk MJ, Blaisdell GY, Wiater BP et al. Traumatic dural tears: What do we know and are they a problem? Spine J 2014; 14: 49-56

10 Mokri B. Spontaneous low pressure, low CSF volume headaches: Spontaneous CSF leaks. Headache 2013; 53: 1034-1053

11 Schievink WI. Stroke and death due to spontaneous intracranial hypotension. Neurocrit Care 2013; 18: 248-251

12 Lee SE, Chung CK, Jahng TA et al. Dural tear and resultant cerebrospinal fluid leaks after cervical spinal trauma. Eur Spine J 2014; 23: 17721776

13 Oertel MF, Korinth MC, Reinges MH et al. Pathogenesis, diagnosis and management of pneumorrhachis. Eur Spine J 2006; 15: 636-643

14 Rahman M, Bidari SS, Quisling RG et al. Spontaneous intracranial hypotension: Dilemmas in diagnosis. Neurosurgery 2011; 69: 4-14; discussion 14

15 Khemka S, Mearza AA. Isolated sixth nerve palsy secondary to spontaneous intracranial hypotension. Eur J Neurol 2006; 13: 1264 - 1265

16 Sciubba DM, Kretzer RM, Wang PP. Acute intracranial subdural hematoma following a lumbar CSF leak caused by spine surgery. Spine (Phila Pa 1976) 2005; 30: E730-E732

17 Chaves C, Freidberg SR, Lee $G$ et al. Cerebral vasospasm following intracranial hypotension caused by cerebrospinal fluid leak from an incidental lumbar durotomy. Case report. J Neurosurg 2005; 102: 152 - 155

18 Thomke F, Bredel-Geissler A, Mika-Gruttner A et al. Spontaneous intracranial hypotension syndrome. Clinical, neuroradiological and cerebrospinal fluid findings. Nervenarzt 1999; 70: 909-915

19 Hadizadeh DR, Kovacs A, Tschampa H et al. Postsurgical intracranial hypotension: Diagnostic and prognostic imaging findings. Am J Neuroradiol 2010; 31: 100-105

20 Andrews RT, Koci TM. Cerebellar herniation and infarction as a complication of an occult postoperative lumbar dural defect. Am J Neuroradiol 1995; 16: 1312-1315

21 Bloch J, Regli L. Brain stem and cerebellar dysfunction after lumbar spinal fluid drainage: Case report. J Neurol Neurosurg Psychiatry 2003; 74: $992-994$

22 Parpaley $Y$, Urbach $H$, Kovacs A et al. Pseudohypoxic brain swelling (postoperative intracranial hypotension-associated venous congestion) after spinal surgery: Report of 2 cases. Neurosurgery 2011; 68: E277-E283

23 Oudeman EA, Nandoe Tewarie RD, Jobsis GJ et al. Complications corner: Anterior thoracic disc surgery with dural tear/CSF fistula and lowpressure pleural drain led to severe intracranial hypotension. Surg Neurol Int 2015; 6: S137-S139

24 Grahovac G, Vilendecic M, Chudy D et al. Nightmare complication after lumbar disc surgery: Cranial nontraumatic acute epidural hematoma. Spine (Phila Pa 1976) 2011; 36: E1761 -E1764

25 Van Roost $D$, Thees $C$, Brenke $C$ et al. Pseudohypoxic brain swelling: A newly defined complication after uneventful brain surgery, probably related to suction drainage. Neurosurgery 2003; 53: 1315-1326; discussion 1326-1327

26 Scholsem M, Scholtes F, Belachew $S$ et al. Acquired tonsillar herniation and syringomyelia after pleural effusion aspiration: Case report. Neurosurgery 2008; 62: E1172 -E1173; discussion E1173
27 Loya JJ, Mindea SA, Yu $\mathrm{H}$ et al. Intracranial hypotension producing reversible coma: A systematic review, including three new cases. J Neurosurg 2012; 117: 615-628

28 Gelalis ID, Karageorgos A, Arnaoutoglou C et al. Traumatic pneumorrhachis: Etiology, pathomechanism, diagnosis, and treatment. Spine J 2011; $11: 153-157$

29 Medina JH, Abrams K, Falcone S et al. Spinal imaging findings in spontaneous intracranial hypotension. Am J Roentgenol 2010; 195: 459-464

30 Caranci F, Briganti F, La Porta $M$ et al. Magnetic resonance imaging in brachial plexus injury. Musculoskelet Surg 2013; 97: S181 - S190

31 Sporns PB, Fortmann T, Zimmer S et al. Traumatische Plexusläsion mit zervikothorakalen Wurzelausrissen und vertikaler Lazeration des Myelons. Fortschr Röntgenstr 2015; 187: 938 -939

32 Chhabra A, Thawait GK, Soldatos T et al. High-resolution 3T MR neurography of the brachial plexus and its branches, with emphasis on 3D imaging. Am J Neuroradiol 2013; 34: 486 - 497

33 Soldatos T, Andreisek G, Thawait GK et al. High-resolution 3-T MR neurography of the lumbosacral plexus. Radiographics 2013; 33: 967-987

34 Schievink WI. Spontaneous spinal cerebrospinal fluid leaks and intracranial hypotension. JAMA 2006; 295: 2286-2296

35 Mokri B. Cerebrospinal fluid volume depletion and its emerging clinical/imaging syndromes. Neurosurg Focus 2000; 9: e6

$36 \mathrm{Huh}$ J. Burr hole drainage: Could be another treatment option for cerebrospinal fluid leakage after unidentified dural tear during spinal surgery? J Korean Neurosurg Soc 2013; 53: 59-61

37 Jung YY, Ju CI, Kim SW. Bilateral subdural hematoma due to an unnoticed dural tear during spine surgery. J Korean Neurosurg Soc 2010; 47: $316-318$

38 Lourenco Costa B, Shamasna M, Nunes J et al. Cerebral venous thrombosis: An unexpected complication from spinal surgery. Eur Spine J 2014; 23: $253-256$

39 Ruiz-Sandoval JL, Campos A, Romero-Vargas S et al. Multiple simultaneous intracerebral hemorrhages following accidental massive lumbar cerebrospinal fluid drainage: Case report and literature review. Neurol India 2006; 54: 421 - 424

40 Lau D, Lin J, Park P. Cranial nerve III palsy resulting from intracranial hypotension caused by cerebrospinal fluid leak after paraspinal tumor resection: Etiology and treatment options. Spine J 2011; 11: e10-e13

41 Khurana A, Brousil J, Russo A et al. Intracranial hypotension with a sixth cranial nerve palsy subsequent to massive thoracic CSF hygroma: A rare complication of thoracic disc excision. Eur Spine J 2013; 22: $2047-2054$

42 Kundu A, Sano Y, Pagel PS. Case report: Delayed presentation of postural headache in an adolescent girl after microscopic lumbar discectomy. Can J Anaesth 2008; 55: $696-701$

43 Albayram S, Ulu MO, Hanimoglu $\mathrm{H}$ et al. Intracranial hypotension following scoliosis surgery: Dural penetration of a thoracic pedicle screw. Eur Spine J 2008; 17: S347-S350

44 Bobbio A, Hamelin-Canny E, Roche $N$ et al. Abducens nerve palsy after schwannoma resection. Ann Thorac Surg 2015; 99: 694-695

45 Nowak R, Maliszewski M, Krawczyk L. Intracranial subdural hematoma and pneumocephalus after spinal instrumentation of myelodysplastic scoliosis. J Pediatr Orthop B 2011; 20: $41-45$

46 Thomas $R$, Thanthulage $S$. Intracranial hypotension headache after uncomplicated caudal epidural injection. Anaesthesia 2012; 67: 416-419 